\section{Is progesterone a neutral or protective factor for breast cancer?}

\author{
Paola Muti
}

In her Review (Progesterone signalling in breast cancer: a neglected hormone coming into the limelight. Nature Rev. Cancer 13, 385-396 (2013)) $)^{1}$, Cathrin Brisken gave an overview of the role of progesterone in breast cancer aetiology. She collected a range of experimental and observational data supporting the hypothesis that increased levels of endogenous progesterone might represent a risk factor for breast cancer and that progesterone receptor activation promotes breast carcinogenesis. The Review was highly appreciated and included many interesting study results. However, some important evidence that supported the opposite hypothesis (that progesterone is a neutral or protective factor for breast cancer) was not included. Here, I wish to list the contradictory evidence in order to provide comprehensive information on the functional activity of progesterone in breast cancer development.

\section{Experimental evidence}

The Review ${ }^{1}$ reports experimental animal data indicating that progesterone is a determinant factor for mammary tumours. However, the paper does not mention other experimental studies showing that progesterone actually reduces oestrogen-induced breast cancer cell proliferation ${ }^{2-5}$.

\section{Observational evidence}

In general, hormonal measurements in premenopausal women (in whom progesterone can be accurately measured only during the luteal phase of the ovarian cycle) are difficult to interpret because of both the variable serum concentrations over the menstrual cycle and the large variability of cycle length within and between individuals. The correlation between repeated measurements of serum sex hormones in the same women is in fact much lower before than after the menopause. In spite of these methodological limitations, the European Prospective Investigation on Cancer and Nutrition (EPIC), which is one of the largest prospective cohort studies on cancer aetiology, investigated the association between sex steroid hormones and breast cancer occurrence in premenopausal women ${ }^{6}$. In that study, the level of serum progesterone was measured in serum samples from 285 premenopausal women who subsequently developed breast cancer (case patients) and from 555 matched cancer-free control subjects who had provided information about the day of menstrual cycle at blood donation. The study observed a statistically significant inverse relationship between serum levels of progesterone and breast cancer incidence. The study replicated the results of the first 7-year follow-up of the ORDET (Hormones and Diet in the Etiology of Breast Cancer) prospective study ${ }^{7}$, which is a smaller study in which sources of technical and biological variability are carefully controlled by study design.

Since the publication of Dr Brisken's Review, two further analyses have been published. The first one is a recent analysis of the 20-year follow-up of the ORDET prospective study, in which the investigators did not observe any risk associated with progesterone serum levels ${ }^{8}$. The second published report describes the pooled analysis of the seven largest prospective cohort studies on hormones and breast cancer risk 9 . The analysis was carried out through the follow-up of many thousands of healthy premenopausal women who provided blood samples. The analysis also confirmed the lack of association between progesterone levels and breast cancer risk.

\section{Hormonal intervention}

The Review correctly reports results from studies on hormonal replacement therapy (HRT) conducted in France. In that country, postmenopausal women usually receive natural progesterone in their HRT composition instead of synthetic progestins. Results from these studies showed that there was no risk effect associated with natural progesterone intake. However, the Review did not adequately discuss that specific evidence.

In conclusion, the role of progesterone in breast cancer development has to be better clarified before considering specific strategies that target it for breast cancer prevention.

Paola Muti is at the Faculty of Health Sciences, 711 Concession Street, G (60) Wing, 1st Floor Room 125, Hamilton, Ontario L8V 1C3, Canada. e-mail:muti@mcmaster.ca doi: 10.1038/nrc3518-c1

1. Brisken, C. Progesterone signalling in breast cancer: a neglected hormone coming into the limelight. Nature Rev. Cancer 13, 385-396 (2013).

2. Foidart, J. M. et al. Estradiol and progesterone regulate the proliferation of human breast epithelial cells. Fertil. Steril. 69, 963-969 (1998).

3. Alkhalaf, M., El-Mowafy, A. \& Karam, S. Growth inhibition of MCF-7 human breast cancer cells by progesterone is associated with cell differentiation and phosphorylation of Akt protein. Eur. J. Cancer Prev. 11, 481-488 (2002).

4. Alkhalaf, M. \& El-Mowafy, A. M. Overexpression of wild-type 53 gene renders MCF- 7 breast cancer cells more sensitive to the antiproliferative effect of progesterone. J. Endocrinol. 179, 55-62 (2003).

5. Lin, V. C., Eng, A. S., Hen, N. E., Ng, E. H. \& Chowdhury, S. H. Effect of progesterone on the invasive properties and tumor growth of progesterone receptortransfected breast cancer cells MDA-MB-231. Clin. Cancer Res. 7, 2880-2886 (2001).

6. Kaaks, R. et al. Serum sex steroids in premenopausal women and breast cancer risk within the European Propsective Investigation into Cancer and Nutrition (EPIC). J. Natl Cancer Inst. 97, 755-765 (2005).

7. Micheli, A. et al. Endogenous sex hormones and subsequent breast cancer in premenopausal women. Int. J. Cancer 112, 312-318 (2004).

8. Schernhammer, E. S. et al. Endogenous sex steroids in premenopausal women and risk of breast cancer: the ORDET cohort. Breast Cancer Res. 15, R46 (2013).

9. Endogenous Hormones and Breast Cancer Collaborative Group. Sex hormones and risk of breast cancer in premenopausal women: a collaborative reanalysis of individual participant data from seven prospective studies. Lancet Oncol. 14, 1009-1019 (2013).

Competing interests statement

The author declares no competing interests. 